\title{
Functionalized Optical Fibre Sensors for Real-Time Environmental Monitoring Applications
}

\author{
Kin Kee Chow \\ School of Engineering \\ Manchester Metropolitan University \\ John Dalton Building, Chester Street \\ Manchester M1 5GD United Kingdom \\ +44 (0) 1612471632 \\ K.Chow@mmu.ac.uk
}

\begin{abstract}
The role of optical fibres as a medium for information transfer has found many applications. One of these is in environmental sensing, where slight perturbations in the ambient environment can be remotely detected through modulation of light at selected points along the optical fibre. These perturbations exist in the form of variations in refractive index (RI), temperature or strain. This paper reviews our recent work on optical fibre-based RI sensors, in particular long period fibre gratings (LPFGs), integrated with carbon nano-materials. These carbon nano-materials with their unique properties modified the sensing scheme of the conventional fibre sensors, allowing them to gain immunity from certain free spectral range limitations, extend their operation range and also present the potential for efficient real-time environmental monitoring applications.
\end{abstract}

\section{Keywords}

Environmental sensors; Fibre-optics devices; Remote sensing

\section{INTRODUCTION}

Environmental sensors have many quality control applications in various industries and water treatment plants. In particular, clean water is crucial for the survival of many of the Earth's living organisms. However, with the advent of industrialization in the world today, water can knowingly or unknowingly be contaminated. Apart from that, it can also serve as a transport medium for certain viruses, which have the potential to create outbreaks or even epidemics. As such, efficient treatment and detection of contaminants in water is required $[1,2]$. This is especially crucial for a country where water is not readily available and various planning and management policies have been put in place to sustain the demand for water. These sources of water are either recycled, imported or from natural sources and have to be checked for their purity and any contaminants before they can be piped to the masses.

One good measure of the purity of water is its refractive index (RI), where the RI of a particular solution can generally be measured by a refractometer. Conventional refractometers can be calibrated to measure the RI and thus the purity of water. However, most of them are large and bulky due to the optical components that they use. As such, real-time and continuous monitoring is not possible and samples of water have to be obtained and transported to a laboratory for measurements of their RI. This is often time consuming and posts a lower limit on the processing time. The more compact and portable refractometers still have to be transported to and from the site, and due to their compactness, have a lower sensing resolution.

On the other hand, optical fibres can be modified at certain areas along their length or at the ends to form sensing probes. Light guided through an optical fibre can respond to various physical, chemical or biological perturbations in the immediate environment of these modified regions (ambient environment), to produce an output in relation to these perturbations [3]. Apart from that, in what is referred to as multiplexing, several of these sensing probes can be located along a single or several optical fibre cables to form a large array of sensors which can be monitored from a remote location [4]. This coupled with certain inherent advantages over their conventional and electronic counterparts allow optical fibres to potentially be applied as real-time and continuous detectors in water treatment plants as well as industrial processes with RI specific requirements.

This paper reviews our recent work on water quality monitoring system. A particular focus will be put on the RI sensing utilizing specialty optical fibre sensors coated with carbon nanotubes (CNTs), which can be further functionalized for different measurands. The sensing mechanism and the fabrication of the CNT-deposited long period fibre grating (LPFG) would be presented. This would be followed by a discussion of the behavior of the spectral features of its output to variations in RI of the ambient environment. A discussion of the sensing performance, repeatability and stability of the sensing probe will be provided.

\section{SENSING MECHANISM}

A LPFG in general is a single mode fibre (SMF) with a periodic modulation in refractive index (RI) in a short segment of the fibre core. Unlike common Bragg grating, the period of modulation is usually in the order of $100 \mu \mathrm{m}$ to $1 \mathrm{~mm}$. The sensitivity of the LPFG to perturbations in its ambient environment, is largely dependent on the differential propagation of its core mode $\left(\mathrm{LP}_{01}\right)$ and forward propagating cladding modes $\left(\mathrm{LP}_{0 \mathrm{~m}}\right)$ in the cladding. Assuming a symmetric fiber with a symmetric grating, as light propagating through the core of the SMF is perturbed by the grating, energy from the $\mathrm{LP}_{01}$ mode would be coupled to the $\mathrm{LP}_{0 \mathrm{~m}}$ modes. The schematic of the CNT-deposited LPFG sensing probe and the light propagation mechanism is shown in Fig. 1. The CNT coating can be treated as an additional dielectric overlay of finite thickness with a RI higher than that of the cladding of the LPFG and the behavior of the LPFG would be similar to that when it is in a medium of higher RI. In this case, the sensing probe can be modelled as a cylindrical waveguide with four layers where the LP modes, crosscoupling coefficients and self-coupling coefficients can be calculated. From here, assuming that there is no other azimuthal variation in RI of the fibre profile, the power in each mode can be expressed as [5]:

$$
p_{0 n}=\frac{\beta_{0 n}}{2 \omega \mu_{0}} \int_{r=0}^{2 \pi} d \phi \int_{r=0}^{r_{\text {core }}} R_{0 n}^{2}(r) d r, n=1,2,3 \ldots
$$




$$
\begin{aligned}
& \omega=k_{0} c \\
& k_{0}=\frac{2 \pi}{\lambda}
\end{aligned}
$$

where $\beta_{0 n}$ is the propagation constant of the $n^{\text {th }}$ LP mode, $\mu_{0}$ is the permeability of free space, $r_{\text {core }}$ is the radius of the fibre core, $\phi$ is the azimuthal angle and $R(r)$ is the radial variation of the modal field. $c$ is a constant defined as the speed of light in vacuum and $\lambda$ is the free space wavelength. From Fig. 1, at the claddingCNT interface, a portion of the power in the cladding modes (dashed arrow) would be multiply reflected and transmitted (dotted arrows) [6]. From here, a variation in the magnitude of the attenuation bands in the output spectrum of the CNT-deposited LPFG would be expected as the RI of the ambient environment varied, where sensing capabilities can be obtained.

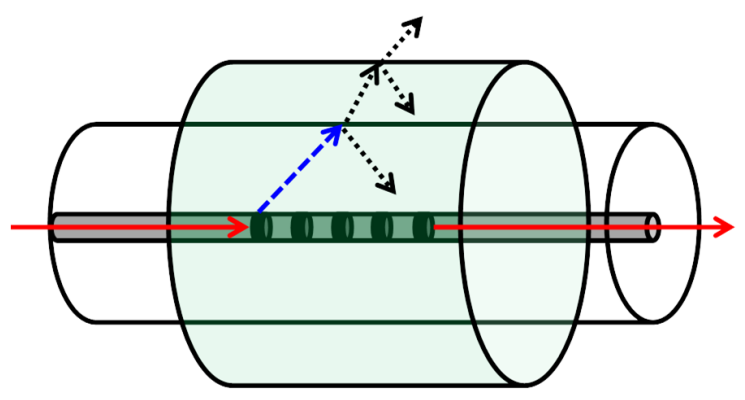

Fig. 1 Schematic of light propagation mechanism of a CNT-deposited LPFG for sensing applications.

\section{FABRICATION OF SPECIALTY FIBRE SENSORS}

In order to prepare the LPFG, the amplitude mask technique using an excimer laser (Lambda Physik) was used to inscribe the long period grating into the core of a SMF. To increase its photosensitivity, the SMF was hydrogen loaded for 10 days before the grating inscription. As shown in Fig. 2, an amplitude mask was placed at a pre-calibrated distance from the hydrogen loaded optical fibre and a Krypton Fluoride $(\mathrm{KrF})$ laser with a laser output wavelength of $248 \mathrm{~nm}$ was scanned normally across the amplitude

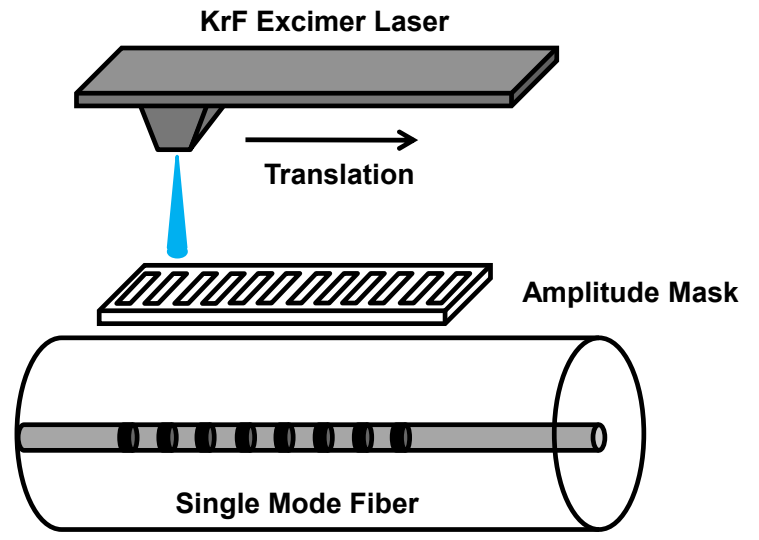

Fig. 1 Schematic of the grating inscription process. mask. The laser beam was diffracted into several beams by the amplitude mask and the constructive and destructive interference of these beams on the fibre core resulted in the formation of a periodic RI pattern in the fibre core [7].

The spray coating method is then applied for CNT deposition onto the fabricated LPFG, which is one of the simplest methods of depositing nano-material overlay over a substrate [8-10]. The setup for depositing the carbon nanomaterials over an optical fibre sensing probe (LPFG) is shown in Fig. 3. The fabricated LPFG, connected in a transmissive configuration, is connected at one end to a white light source and an optical spectrum analyser (OSA) at the other end. The purpose of the light source and OSA is to monitor any variations in the observable spectral features throughout the deposition process and also to ensure that these spectral features are observable throughout the deposition process, as these spectral features would serve as crucial reference points during the RI sensing process.

The LPFG was held straight over a silicon or glass substrate the purpose of which was to characterize the thickness of the deposited carbon nanomaterial. A spray gun (Harder and Steenbeck, Evolution Two in One) was connected to a nitrogen gas supply, and the pressure of the gas flowing into the spray gun was set at 5 Psi. A small volume of carbon nanomaterial solution was transferred into the solution cup connected to the spray gun. The nozzle of the spray gun was then aligned directly over the sensing probe. To deposit the carbon nanomaterials, a valve would be released and nitrogen gas would be pumped into the spray gun and disperse the carbon nanomaterials over the sensing probe via the nozzle. The spray gun was moved in a horizontal direction along the length of the sensing probe to ensure that the entire sensing probe was coated with the carbon nanomaterials. The fibre was rotated after each spray to ensure that the carbon nanomaterials were evenly deposited on the entire surface of the sensing probe.

One important point to note about this method is that although the various parameters like gas pressure and spray duration can be controlled to give a homogeneous deposited layer, it is very difficult to ensure that the homogeneity extends over the entire surface. Hence the thickness of the deposited carbon nanomaterial has to be measured by simultaneously spraying a silicon or glass wafer and estimating the thickness from the scanning electron microscope image of the wafer.

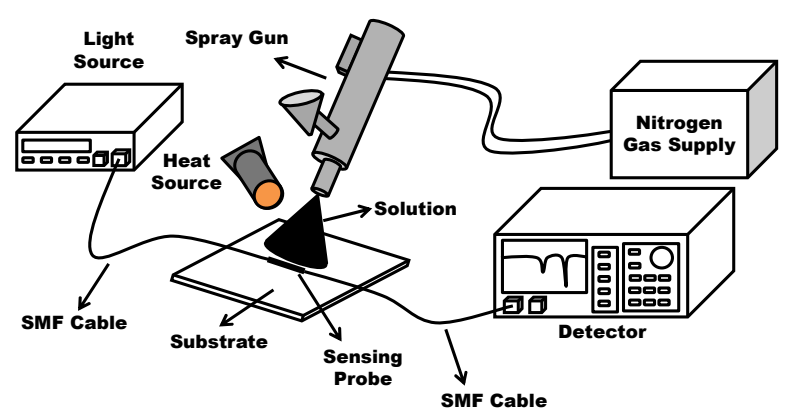

Fig. 3 Schematic of the setup for spray coating of carbon nanomaterials over an optical fibre sensing probe (LPFG).

The optical microscope images focusing on the LPFG region before and after deposition of the CNTs are shown in Fig. 4. Note that the CNTs were evenly deposited over the entire surface of the LPFG. 
From here the thickness of the CNT coating was estimated to be approximately a few micrometers. Fig. 5 shows the output transmission spectrum of the CNT-deposited LPFG. The output spectrum before and after deposition remained relatively the same with only a decrease in magnitude of the attenuation band at a wavelength of $1463.2 \mathrm{~nm}$ after deposition. This can be explained by treating the CNT-deposited LPFG as a LPFG surrounded by a medium with a higher RI as described in the previous section. The high RI of CNT resulted in the coupling of energy from the core modes to the radiation modes resulting in a variation in the magnitude of the intensity of the attenuation band.

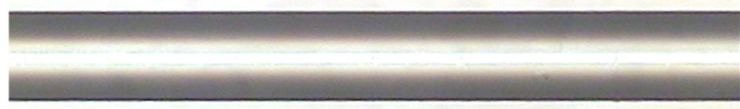

(a)

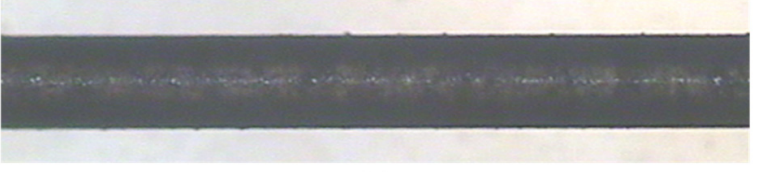

(b)

Fig. 4 Optical microscope image of the LPFG (a) before and (b) after deposition of CNTs.

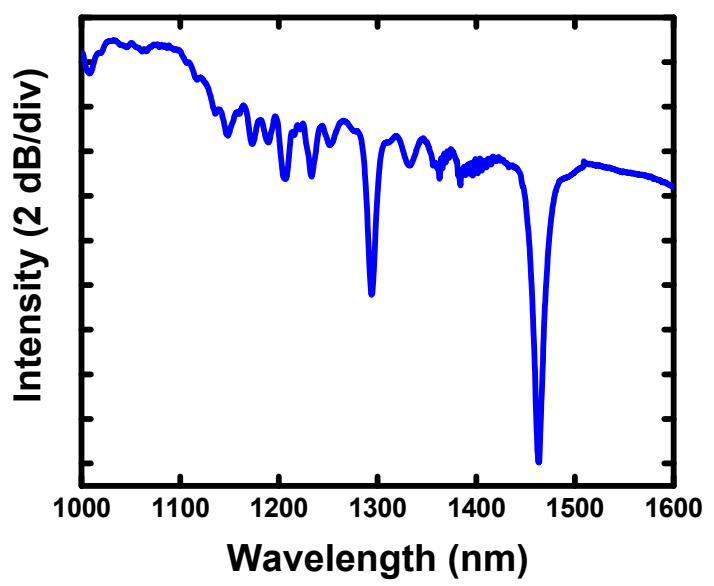

Fig. 5 Output transmission spectrum of the CNTdeposited LPFG.

\section{SENSING PERFORMANCE AND RESULTS}

The CNT-deposited LPFG was connected to a white light source and an OSA in the transmissive configuration. The RI characterization procedure was carried out with the sensing element immersed in different RI solution. The behavior of the CNTdeposited LPFG to variations in RI of the ambient environment is shown in Fig 6. The behavior to the range RIs shown in the figure was obtained continuously with no need for any reference points to be taken. From the figure, it can be seen that there were clear and distinct variations in the magnitude of the intensity of the attenuation band at $1463.2 \mathrm{~nm}$. The slight ripple-like fluctuations in the attenuation band can be attributed to the light source and can be overcome by using a more stable light source. As can be seen, the magnitude of the intensity of the attenuation band decreased as the RI of the ambient environment increased.

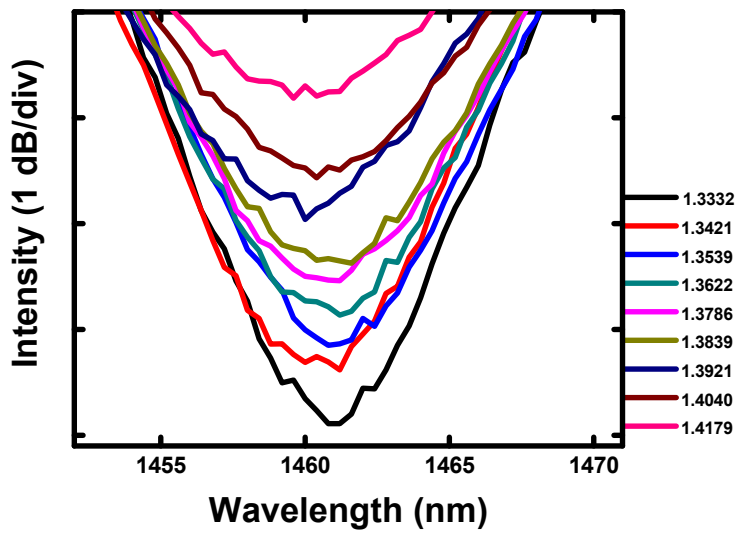

Fig. 6 Behavior of the attenuation band at $1463.2 \mathrm{~nm}$ to variations in $\mathrm{RI}$ of the ambient environment.

The variation in wavelength in relation to variations in RI of the ambient environment is shown in Fig. 7. The wavelength remained relatively constant, deviating within the wavelength range of $1460.80-1463.2 \mathrm{~nm}$ as the RI of the ambient environment varied. This deviation was insufficient to draw any conclusions on the encoding of RI variations of the ambient environment in the wavelength. The slight fluctuations observed can be attributed to errors from the detector and also the strain on the LPFG caused by minor differences in the volume of each test solution used. It can be seen that there is little wavelength dependence of the attenuation band with RI of the ambient environment implying that the variation in RI of the ambient environment can be solely encoded by the intensity variations of the attenuation band.

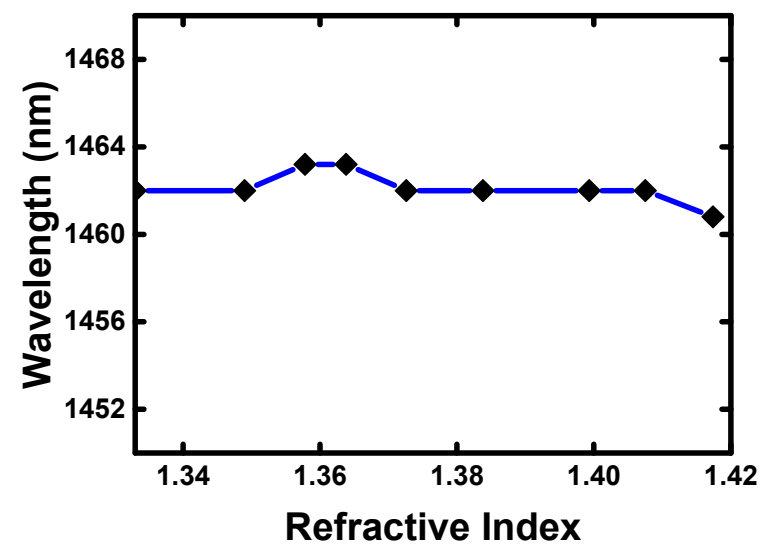

Fig. 7 Variation in wavelength of the attenuation band at $1463.2 \mathrm{~nm}$ to variations in RI of the ambient environment.

In order to further test the repeatability of the sensor, a second RI characterization trial was carried out. The variations in intensity of the attenuation band of both trials (diamonds for the first trial, circles for the second trial) were normalized and are shown in Fig. 8. As can be seen, the change in intensity for both trials increased as the RI of the ambient environment increased. A single fitting line was plotted through the experimental data from both trials and it 
can be seen that there is a nonlinear relation between the intensity of the attenuation band and the RI of the ambient environment. The achieved sensitivity was calculated to be approximately $31 \mathrm{~dB} / \mathrm{RIU}$ and $47 \mathrm{~dB} / \mathrm{RIU}$ for the RI ranges of 1.33-1.38 and 1.38-1.42, respectively. Based on these values, the minimum achievable resolution for the CNT-deposited LPFG for the RI range of 1.331.38 was calculated to be approximately $3.23 \times 10^{-4}$. The maximum error due to the repeatability was calculated to be approximately $0.4 \mathrm{~dB}$ and this error can be attributed to fluctuations from the source and errors from the detector. These results indicate that the sensor has good repeatability over time and is capable of demonstrating distinct variations in intensity as the RI of the ambient environment varied.

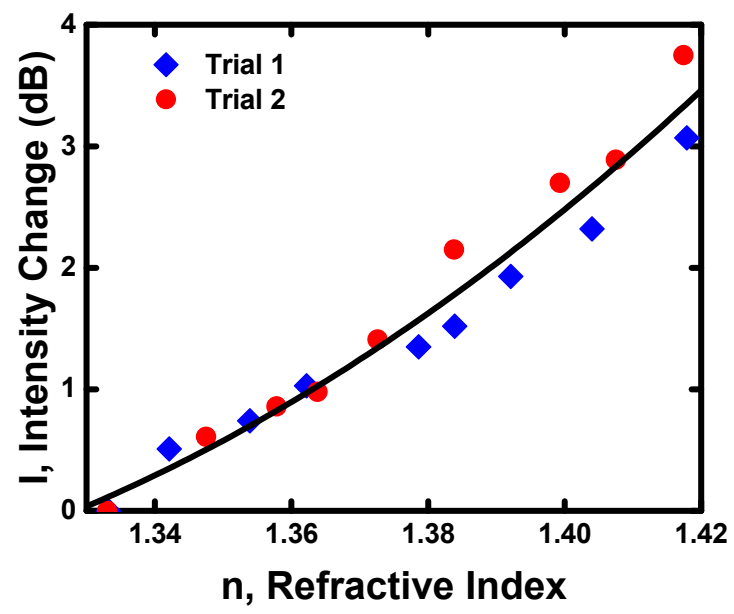

Fig. 8 Variation in intensity of the attenuation band at $1463.2 \mathrm{~nm}$ to variations in RI of the ambient environment for two separate trials.

From the results, it can be seen that the variation in RI of the ambient environment can be solely encoded in the variation in intensity of the attenuation band in the output spectrum of the CNTdeposited LPFG. This modification in sensing scheme would allow the CNT-deposited LPFG to encode a secondary parameter through variations in wavelength of the attenuation band. The CNTdeposited LPFG has also shown good repeatability tests with no conclusive degradation in sensing performance.

The sensitivity of the CNT-deposited LPFG is also believed to be able to further improve. The attenuation band that was chosen was at a wavelength position far from the telecommunications wavelength which would lead to problems related to power losses. One of the ways to overcome this issue is to use LPFGs with a shorter grating period $\Lambda$. Gratings with shorter periods would have their higher order resonance bands, and thus attenuation bands, blue shifted to lower wavelengths to within the detectable region. These higher order resonance bands have been shown to exhibit greater sensitivity and their blue shifting to wavelengths in the detectable range of the OSA (or power meter) would enable their utility for better sensitivity performance of the sensing probe.

\section{SUMMARY}

In summary, the behavior of the CNT-deposited LPFG to variations in RI of the ambient environment has been presented. The sensitivity and repeatability have also been discussed. Through the modification of the sensing scheme, the sensing capabilities of the CNT-deposited LPFG have been enhanced further. Applications into chemical sensing would also be explored. It has been shown that CNTs can be functionalized to allow certain molecules to bind to functionalized sites on the CNTs [11]. By functionalizing the CNTs before deposition, various analyte specific optical fibrebased sensing probes can be fabricated, opening up the possibility for applications in chemical sensing. Also, the multiplexing capabilities of the fibre sensing probes would also be explored. This would involve the arraying of the sensing probes into a sensing network using wavelength division or time division multiplexing techniques to monitor a large area or several areas localized simultaneously.

\section{REFERENCES}

[1] T. G. Aw, K. Y. H. Gin, L. L. E. Oon, E. X. Q. Chen and C. H. Woo, "Emerging contaminants of public health significance as water quality indicator compounds in the urban water cycle," Environ. Int, vol. 71, pp. 46-62, Oct. 2014.

[2] A. K. Biswas, C. Tortajada and R. Izquierdo-Avino, Water Management in 2020 and Beyond: Springer Berlin Heidelberg, 2009.

[3] B. Culshaw, "Optical fibre sensor technologies: opportunities and - perhaps - pitfalls," J. Lightw. Technol., vol. 22, no. 1, pp. 39-50, Jan. 2004.

[4] K. T. V. Grattan and T. Sun, "Fibre optic sensor technology: an overview," Sens. Actuators, A, vol. 82, no. 1-3, pp. 40-61, May. 2000.

[5] Y. Koyamada, "Numerical analysis of core-mode to radiationmode coupling in long-period fibre gratings," IEEE Photon. Technol. Lett., vol. 13, no. 4, pp. 308-310, Apr. 2001.

[6] D. B. Stegall and T. Erdogan, "Leaky cladding mode propagation in long-period fibre grating devices," IEEE Photon. Technol. Lett., vol. 11, no. 3 pp. 343-345, Mar. 1999.

[7] G. P. Agrawal, Applications of nonlinear fibre optics, $2^{\text {nd }}$ ed. Amsterdam, Boston: Academic Press/Elsevier, 2008.

[8] S. Y. Set, H. Yaguchi, Y. Tanaka, and M. Jablonski, "Laser mode locking using a saturable absorber incorporating carbon nanotubes", J. Lightw. Technol., vol. 22, no. 1, pp. 51-56, Jan. 2004.

[9] H. H. Liu, Y. Yang, and K. K. Chow, "Enhancement of thermal damage threshold of carbon-nanotube-based saturable absorber by evanescent-field interaction on fiber end," Opt. Express, vol. 21, pp. 18975-18982, Aug. 2013.

[10] H. H. Liu, K. K. Chow, S. Yamashita, and S. Y. Set, "Carbonnanotube-based passively Q-switched fiber laser for high energy pulse generation," Opt. Laser Technol., vol. 45, pp. 713-716, Feb. 2013

[11] S. Kruss, A. J. Hilmer, J. Q. Zhang, N. F. Reuel, B. Mu and M. S. Strano, " Carbon nanotubes as optical biomedical sensors," Adv. Drug Deliv. Rev., vol. 65, no. 15, pp. 19331950, Dec. 2013. 